\title{
Effect Analysis of Gurson Model Parameters on Crack Extension of Pipeline
}

\author{
Tieping Li, Xinlu Tian \\ Nuclear and Radiation Safety Center of MEP, Beijing, China \\ Email: everlasting_cat@sina.com,xinlu724@163.com
}

Received August 2013

\begin{abstract}
Leakage-before-break technique is widely used in high energy pipeline of nuclear plant, for which crack stability of pipeline under complex loading condition is a key issue, and crack growth resistance curve of pipeline material is the important foundation for crack stability analysis. In this paper, ferritic steel A533B is studied, Gurson damage model is used to simulate crack process of contact tension specimen under uni-tension, and effect of Gurson model parameter on simulation result is discussed. The following results are found during simulation: initial porosity factor $f_{0}$ is the main parameter, when it increases gradually, unstable crack extension will be observed; however, only initial J toughness is affected by critical porosity factor $f_{N}$; the minor parameter is load step control $\alpha$, when it increases, stable and convergent result is obtained. All results in this paper can be used to determine parameters in Gurson mode, which will be foundation for crack extension analysis of pipeline.
\end{abstract}

Keywords: Leakage-Before-Break; Crack Growth Resistance Curve; Gurson Damage Model; Crack Extension

\section{Introduction}

In present, leakage-before-break (LBB) technique is widely used for collant pipeline in most operating and abuilding nuclear plants. In LBB technique, leakage can be detected in pipeline before unstable crack extension occurring in pipeline, and protective hardware and whip restraints can be removed for convenience of inspection operability, from which economic efficiency can be guaranteed. Since austenitic steel with high toughness is used for nuclear pipeline, elastic-plastic fracture is the main failure mode. Elastic-plastic fracture is a complex process, and fracture toughness $J_{I C}$ cannot be adapted directly to fracture analysis in complex structure [1]. Combination of fracture test and material model is proposed by some researchers since fracture mechanism is required by fracture process simulation [2], material model parameters can be deduced from combination result [3], which can be used to simulate fracture process in any complex structure.

Gurson model is proved to simulate fracture process well for metallic material from a lot of tests and theoretic studies [4]. Contact tension specimen made of ferritic steel A533B is studied in this paper, Gurson model is combined to describe micro fracture process in A533B material, and effect of Gurson model parameter on simulation results is analyzed by finite element method.

\section{Gurson Model}

Metallic material is divided into two parts in Gurson model: basement material and porosity. Initial porosity is assumed as $f_{0}$, when material is loaded, bigger porosity is formed by connection of smaller porosity, and new crack surface is obtained at the same time. Material failure can be judged by the following equation:

$$
g=\left(\frac{\sigma_{e}}{\bar{\sigma}}\right)^{2}+2 q_{1} f \cosh \left(\frac{3 q_{2} \sigma_{m}}{2 \bar{\sigma}}\right)-\left(1+q_{3} f^{2}\right)=0
$$

In Equation (1), $\sigma_{e}$ is regarded as equivalent stress of the whole material, $\sigma_{m}$ is hydrostatic pressure, $\bar{\sigma}$ is equivalent stress of basement material, $f$ is real-time porosity rate of the whole material. When $f$ is larger than $f_{N}$, which is defined as the critic porosity, crack extension will be found. In above equation, $q_{1}, q_{2}, q_{3}$, is aspect ratio of porosity, which can be deduced by stress-strain curve of uni-tension test [5].

\section{Simulation Analysis}

\subsection{Material Property}

The stress-strain curve of ferritic steel A533B can be described as the following relationship: 


$$
\varepsilon=\frac{\sigma_{0}}{E}\left(\frac{\sigma}{\sigma_{0}}\right)^{1 / N}
$$

In Equation (2), $\sigma_{0}$ is initial yielding stress of material, $N$ is strain hardening index, $E$ is young's modulus, and for material of A533B, parameters defined in Equation (2) are fixed as $\sigma_{0}=400 M P a, E=2 \times 10^{5} M P a, N=0.1$.

\subsection{Finite Element Model}

According to ASME fracture toughness test standard E1820 [6], the geometric character of contact tension specimen can be plotted as Figure 1: $w$ is $50.8 \mathrm{~mm}$ as width of specimen, $B$ is $127 \mathrm{~mm}$ as depth of specimen, and size of the remanent part can be deduced by the proportion in Figure 1. Geometric size in Figure $\mathbf{1}$ is used to build the finite element model.

Software Warp3d is used here for model creation and element C3D8 is selected. Since plastic deformation is included in simulation, crack tip singularity is not considered in this paper. Plot in Figure $\mathbf{2}$ is the half model of contact tension specimen, one-layer element with Gurson model property is assigned to the crack face, and the elastic-plastic material relationship in Equation (2) is still suitable for the remanent part. In this paper, some parameters such as aspect ratio and Gurson element height are fixed in simulation as $q_{1}=1.46, q_{2}=0.931, q_{3}=2.131$ and $D=200 \mu \mathrm{m}$, where $D$ is the height of element with Gurson model property.

The boundary condition is: all node freedom in depth direction is fixed; node freedom in vertical direction for symmetric surface except crack face is fixed; in order to remove rigid displacement of the whole model, one of the nodes far from loading position and crack surface is

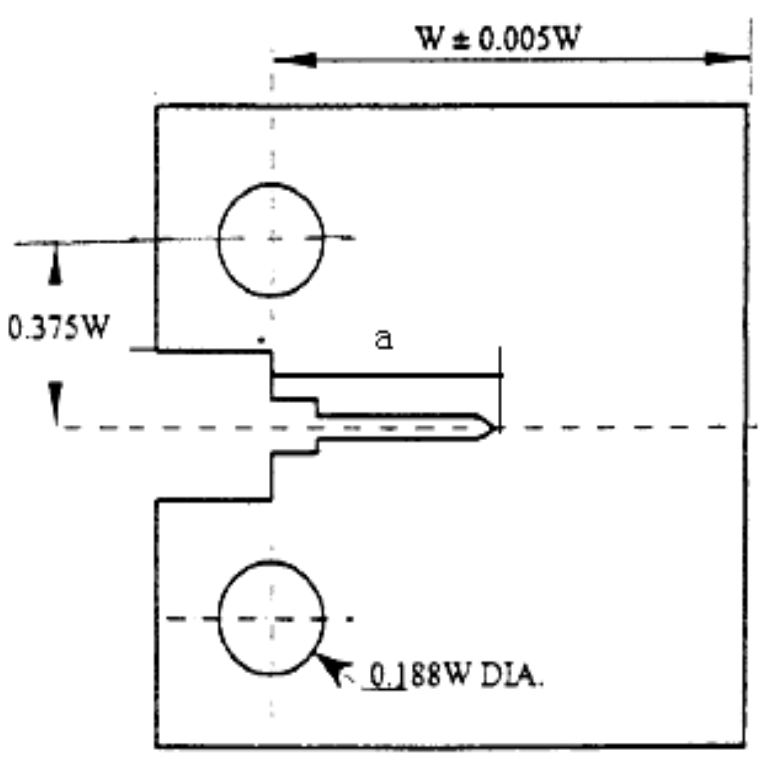

Figure 1. Schematic plot of contact tension specimen.

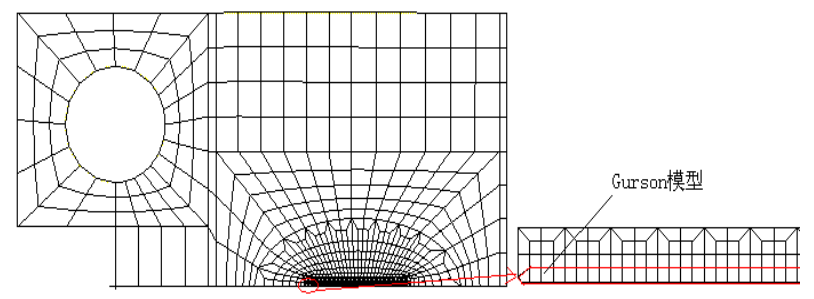

Figure 2. Finite element model of contact tension specimen.

fixed in horizontal direction. Displacement loading method is used.

\subsection{Calculation Results}

Software Warp3d is used to simulate fracture process of contact tension specimen. Effects of initial porosity $f_{0}$, critic porosity $f_{N}$ and load control parameter $\alpha$ embedded in Warp3d on fracture process are studied in simulation.

1) Effect of load control parameter $\alpha$ on $J$ value

For the simulation of this part, initial crack length ratio is $a / w=0.3$, initial porosity rate is $f_{0}=0.005$, critic porosity ratio is $f_{N}=0.2$ and only load control parameter is changeable. It is shown in Figure $\mathbf{3}$ that, curve of $J$ value vs. displacement loading is affected little by load control parameter, and the specimen is perfect now with no crack extension. As displacement $\Delta$ increasing, curves are distinct with different $\alpha$ : smaller $\alpha$ value is related with stable crack extension, such as there is a platform in curve corresponding to $\alpha=0.005$; curves with larger $\alpha$ are related with unstable crack extension since there is a downtrend in these curves.

2) Effect of critic porosity

For the simulation of this part, initial crack length ratio is defined as $a / w=0.5$, load control parameter is defined as $\alpha=0.005$, initial porosity is defined as $f_{0}=0.003$, and only critic porosity $f_{N}$ is changeable. It is shown in Figure 4 that almost no effect of $f_{N}$ on result can be observed.

3) Effect of initial porosity

For the simulation of this part, initial crack length ratio is defined as $a / w=0.5$, load control parameter is defined as $\alpha=0.005$, critic porosity is defined as $f_{N}=0.2$, and only initial porosity $f_{0}$ is changeable. It is shown in Figure 5 that curves of $J$ value vs. displacement loading are coincident with smaller loading. As loading $\Delta$ increases, yielding is found around crack tip since smaller $f_{0} \quad\left(f_{0}=0.001\right)$ corresponding to more perfect material, and there is an uptrend for $J$ value because yielding zone is expanding. As $f_{0}$ increases, there is a downtrend in curve, since material with larger porosity rate is fragile, and relaxative stress can be observed around crack tip with crack surface extension. 


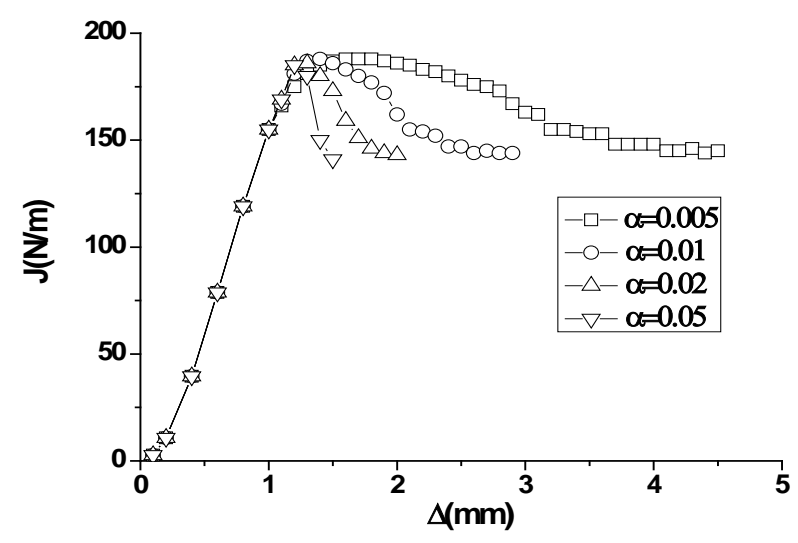

Figure 3. Effect of load-control parameter on calculation results.

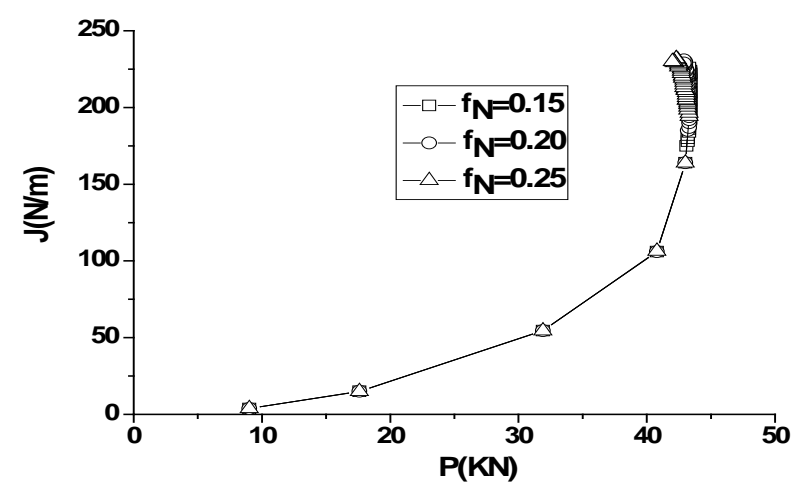

Figure 4. Effect of critic porosity on calculation.

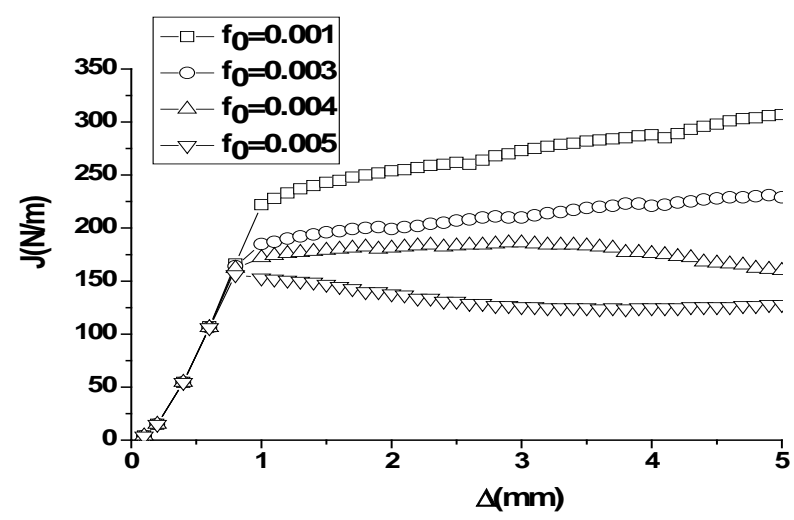

Figure 5. Effect of initial porosity on calculation results.

\section{Conclusions}

The fracture process of contact tension specimen made of ferritic steel A533B is simulated by finite element method combining with Gurson damage model, and effect of parameters in Gurson model on simulation result is studied, from which the following conclusions can be obtained:

1) The initial porosity rate is the leading role for simulation result, when $f_{0}$ increases, there is an unstable trend for crack extension, and only $J$ value in initial loading period is affected by critic porosity rate $f_{N}$.

2) With decreasing load control parameter of $\alpha$, stable and convergent crack extension can be observed.

\section{Acknowledgements}

This work was financially supported by Natural Key Projects of China (Grant No. 2011ZX06002-010).

\section{REFERENCES}

[1] Z. R. Huang and Y. K. You, "Analysis of the Load-Displacement Relationship to Determine the JR Resistance Curve of Carbon Steel Piping,” Journal of Jiangsu Institute Petrochemical Technology, Vol. 12, No. 3, 2000, pp. $1-3$

[2] T. L. Anderson, "Fracture Mechanics: Fundamentals and Applications,” CRC Press, Boca Raton, 1994.

[3] F. Jonas, X. S. Gao and C. F. Shih, "Cell Model for Nonlinear Fracture Analysis: I. Micromechanics Calibration," International Journal of Fracture, Vol. 89, No. 4, 1998, pp. 355-373. http://dx.doi.org/10.1023/A:1007421420901

[4] X. Lin and C. F. Shih, "Ductile Crack Growth I: A Numerical Study Using Computational Cells with Microstructurally-Based Length Scales," Journal of the Mechanics and Physics of Solids, Vol. 43, No. 2, 1995 pp. 233-259.

http://dx.doi.org/10.1016/0022-5096(94)00064-C

[5] “WARP3D-Release 15.9,” University of Illinois, 2008.

[6] ASTM E1820-05a Standard Test Method for Measurement of Fracture Toughness, ASTM International, 2008. 